S83 INVESTIGATING THE ROLE OF GCN2 IN THE PATHOGENESIS OF PULMONARY HYPERTENSION

${ }^{1}$ E Soon, ${ }^{1} \mathrm{~A}$ Crosby, ${ }^{2} \mathrm{M}$ Southwood, ${ }^{1} \mathrm{~S}$ Moore, ${ }^{1} \mathrm{D}$ Ron, ${ }^{1} \mathrm{~S}$ Marciniak, ${ }^{1} \mathrm{NW}$ Morrell. ${ }^{1}$ University of Cambridge, Cambridge, UK; ${ }^{2}$ Papworth Hospital NHS Trust, Cambridge, UK

\subsection{6/thoraxjnl-2016-209333.89}

Background Mutations in the bone morphogenetic protein type II receptor (BMPR-II) underlie the majority $(>80 \%)$ of familial and up to $25 \%$ of 'sporadic' cases of idiopathic pulmonary arterial hypertension (PAH). Recently, homozygous recessive mutations in GCN2 (also known as EIF2AK4) were identified as causative in a rare cause of $\mathrm{PAH}$, pulmonary veno-occlusive disease (PVOD) and pulmonary capillary haemangiomatosis (PCH). The mechanisms by which GCN2 deficiency contributes to the development of PAH remain unknown. GCN2 is a serine/threonine protein kinase, one of a family of 4 kinases that phosphorylate the $\alpha$-subunit of the translation initiation factor eIF $2 \alpha$. Phosphorylation of eIF $2 \alpha$ at serine 51 results in an inhibition of eIF2B (the eIF2 guanine nucleotide exchange factor), which ultimately leads to loss of eIF $2 \alpha$ function. This results in inhibition of protein synthesis while enhancing the translation of a small number of mRNAs encoding proteins involved in the response to cellular stress.

Methods We characterised the pulmonary vascular phenotype of the homozygous null Gcn2 (Gcn2-/-) and wild-type controls at 6 months of age. This mouse was chosen because it mimics the homozygous loss-of-function genotype of patients with familial $\mathrm{PVOD} / \mathrm{PCH}$. In addition, we investigated the phenotype of Gcn2-/- mice crossed with bmpr2-deficient mice. In complementary in vitro studies, pulmonary artery smooth muscle cells (PASMCs) were extracted from patients with PVOD and idiopathic/heritable PAH and studied with wild-type controls.

Findings The Gcn2-/- mouse displayed mild pulmonary hypertension. Mean right ventricular systolic pressure (RVSP) was $28.3 \pm 1.2 \mathrm{mmHg}$ in Gcn2-/- mice compared with $24.7 \pm 1.1$
$\mathrm{mmHg}$ in wild-type mice. GCN2-/- mice crossed with bmpr2deficient mice demonstrated a further increase in RVSP $(32.8 \pm 4.1 \mathrm{mmHg})$. Exposure of human PASMCs derived from a PVOD patient, and patients with mutations in BMPR2, to Lhistidinol, which increases the phosphorylation of eIF $2 \alpha$, resulted in proliferation of these cells but had no effect on control PASMCs.

Conclusions GCN2 deficiency promotes the development of pulmonary hypertension in mice, an effect that is exaggerated by BMPR2 deficiency. Increased phosphorylation of eIF2 $\alpha$ resulted in PASMC proliferation. Further elucidation of these mechanisms may reveal new treatments for this devastating disease.

\section{S84 IDENTIFICATION OF MIR-124A AS A MAJOR REGULATOR OF ENHANCED ENDOTHELIAL CELL GLYCOLYSIS IN PULMONARY ARTERIAL HYPERTENSION}

${ }^{1} \mathrm{P}$ Caruso, ${ }^{1} \mathrm{BJ}$ Dunmore, ${ }^{2} \mathrm{~K}$ Schlosser, ${ }^{3} \mathrm{~S}$ Schoors, ${ }^{2} \mathrm{C}$ Dos Santos, ${ }^{2} \mathrm{C}$ Perez-Iratxeta, ${ }^{2} \mathrm{JR}$ Lavoie, ${ }^{1} \mathrm{~L}$ Long, ${ }^{1} \mathrm{~L}$ Hurst, ${ }^{4} \mathrm{ML}$ Ormiston, ${ }^{5} \mathrm{~A}$ Hata, ${ }^{3} \mathrm{P}$ Carmeliet, ${ }^{2} \mathrm{DJ}$ Stewart, ${ }^{1}$ NW Morrell. ' University of Cambridge, Cambridge, UK; ${ }^{2}$ Ottawa Hospital Research Institute and the University of Ottawa, Ottawa, Canada; ${ }^{3}$ Laboratory of Angiogenesis and Neurovascular Link, Vesalius Research Centre, Leuven, Belgium; ${ }^{4}$ Queen's University, Kingston, Canada; ${ }^{5}$ Cardiovascular Research Institute, University of California, San Francisco, USA

\subsection{6/thoraxjnl-2016-209333.90}

Introduction Pulmonary arterial hypertension (PAH) is a rare desease characterised by profound vascular abnormalities in the peripheral arteries of the lung, leading to a progressive increase in pulmonary vascular resistance, right heart failure and death. The disease exists in several forms including a heritable form (HPAH) caused primarily by mutations in bone morphogenetic protein receptor type 2 (BMPR2) and an idiopathic form (IPAH). Endothelial cell (EC) dysfunction is considered a critical initiating factor in the pathobiology of $\mathrm{PAH}$, manifested by increased

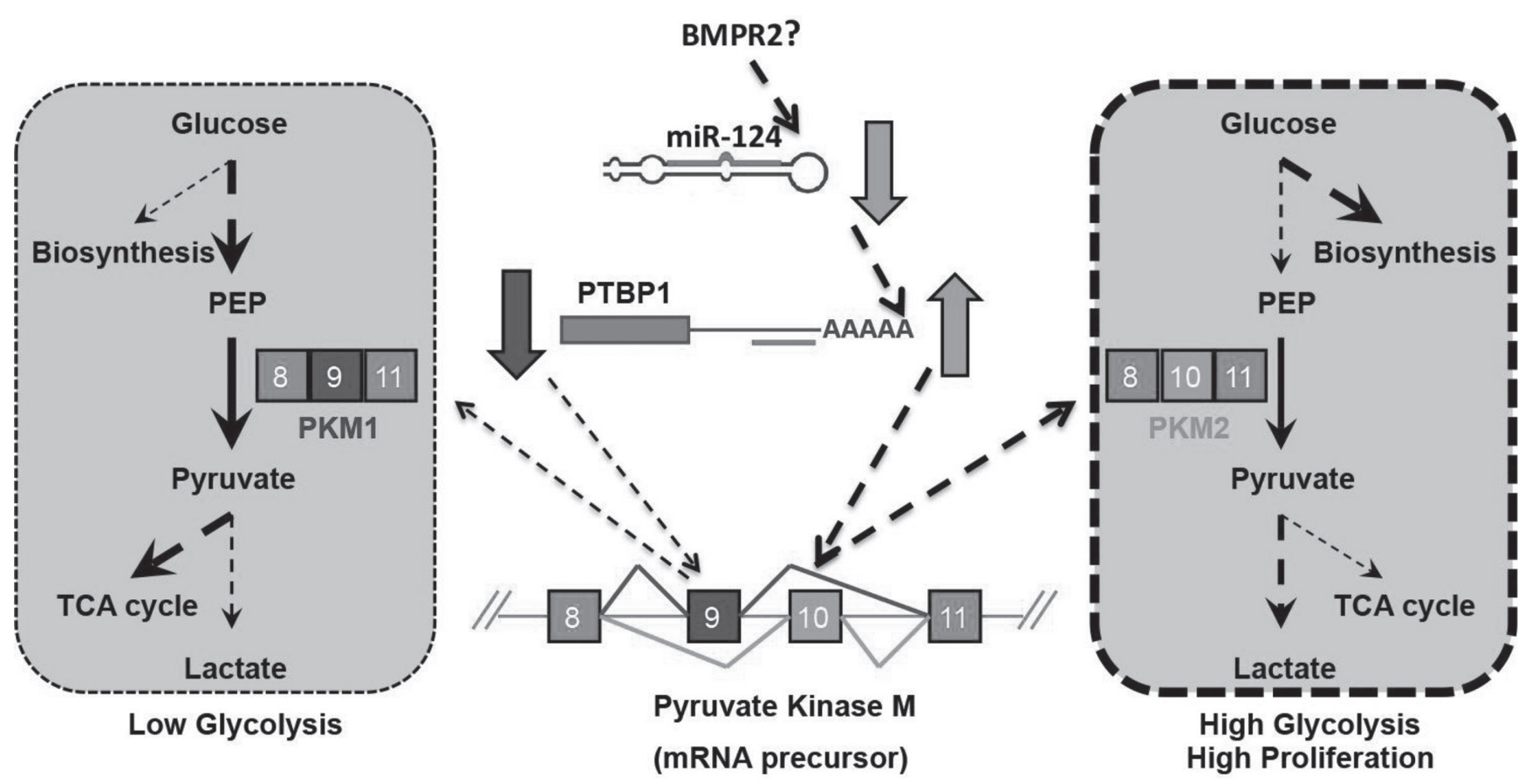

Abstract S84 Figure 1 
susceptibility to apoptosis, heightened permeability and enhanced endothelial proliferation. Substantial changes in bioenergetics of ECs, including higher rates of glycolysis, have been reported in PAH patients. However, the mechanisms underlying alterations in energy production have not been identified.

Methods We measured glycolysis in blood outgrowth endothelial cells (BOECs) from HPAH patients carrying mutations in BMPR2 and IPAH patients to confirm the metabolic abnormalities previously. We also employed an unbiased genome-wide microarray and proteomic screening approach to detect miRNAs and proteins dysregulated in the same groups to determine the mechanisms underlying abnormal endothelial glycolysis.

Results HPAH and IPAH BOECs recapitulated the metabolic phenotype previously observed in PAECs. These alterations were found to be associated with the downregulation of miR-124 and the upregulation of its known target, splicing factor polypyrimidine-tract-binding protein (PTBP1). We also demonstrated that increased PTBP1 promotes the switching in expression of two forms of pyruvate kinase, PKM1 and PKM2, resulting in an increase of aerobic glycolysis, consequently increasing cell proliferation (mechanism schematized in Figure 1). Overexpression of miR-124, or siRNA silencing of PTPB1, restoring normal expression levels of PKM2, also restored normal proliferation and glycolysis in HPAH BOECs. Finally, we observed reduced miR-124 and increased PTPB1 and PKM2 expression in a well-established rat model of $\mathrm{PAH}$, characterised by endothelial proliferation, supporting the presence of this mechanism in vivo.

Conclusions Loss of function of BMPR2 results in the downregulation of miR-124 and consequently in the glycolytic abnormalities reported in PAH ECs. Therefore, the manipulation of this miRNA, or its targets, could represent a novel and effective strategy to achieve clinical benefits in the treatment of PAH.

\section{S85 REDUCTION OF CD68 MACROPHAGES CAUSES GENDER SPECIFIC SPONTANEOUS PULMONARY ARTERIAL HYPERTENSION IN MICE}

A Zawia, N Arnold, A Braithwaite, J Pickworth, K Hopkinson, J Iremonger, G Miller, A Lawrie. The University of Sheffield, Sheffield, UK

\subsection{6/thoraxjnl-2016-209333.91}

Introduction Macrophages are proposed to play an important regulatory role in the pathogenesis of pulmonary arterial hypertension (PAH) as excessive infiltration detected around vascular lesions in patients and animal models. The exact 'causal' role for macrophages, and whether their presence or absence is required for the vascular remodelling seen in $\mathrm{PAH}$ remains unclear.

Objectives Using a novel inducible macrophage depletion model (MacLow mouse) we aimed to determine the role of macrophages in pulmonary arterial remodelling associated with PAH.

Methods Macrophage depletion was induced in MacLow mice by administration of doxycycline, where macrophage-specific induction of the cytotoxic diphtheria toxin A chain (DTA) is driven by the CD68 promoter. Mice were phenotyped for PAH by echocardiography, closed chest cardiac catheterization and immunohistochemistry (IHC) after 6 weeks. To investigate the origin of the effector cells, male chimeric mice were generated, and the disease stimulated by inducing macrophage ablation with doxycycline. Furthermore, to study gender-specificity of the disease phenotype, MacLow mixed gender chimeric mice were produced, and macrophage ablation induced as previous.
Results Interestingly male but not female MacLow mice developed a PAH phenotype compared to controls (RVSP of 66.1 $\mathrm{mmHg}$ vs $24.5 \mathrm{mmHg}, \mathrm{p}<0.0001, \mathrm{n}=5-8$ ), associated with increased right ventricular Hypertrophy (RVH 0.264 vs 0.226 , $\mathrm{p}<0.001, \mathrm{n}=8$ ) and pulmonary vascular remodelling. IHC analysis of diseased lungs demonstrated increased iNOS- |CD206 $+\mid \mathrm{F} 4 / 80+$ macrophages suggesting a M2-like macrophage population drive the PAH phenotype in these mice. The bone marrow transplant studies shows that bone marrow (BM) derived cells contribute in the development of the disease phenotype as wild type BM cells attenuate disease progression. Moreover, female BM transplanted into male mice alleviate but does not protect them from developing PAH.

Conclusion Development of PAH in male MacLow mice suggests that macrophages play a causal role in pulmonary vascular remodelling. Results suggest that the phenotype is driven by lung resident M2- like macrophages with a contribution from bone marrow derived cells. A study to examine the probable protective effect of Oestrogen is now underway to further investigate the implication of gender difference in the incidence of $\mathrm{PAH}$ in this model.

\section{S86 UNDERSTANDING THE ROLE OF GCN2 IN MODULATING BMP SIGNALING USING DROSOPHILA - IMPLICATIONS FOR PULMONARY HYPERTENSION}

E Malzer, CS Dominicus, SJ Marciniak. University of Cambridge, Cambridge, UK

\subsection{6/thoraxjn--2016-209333.92}

Introduction and objectives The majority of inherited cases of pulmonary arterial hypertension (PAH) are caused by mutations that affect the Bone Morphogenetic Protein (BMP) receptor 2. Recently, patients with pulmonary veno-occlusive disease (PVOD), a rare subtype of pulmonary hypertension, were found to have mutations of EIF2AK4. This gene encodes the kinase GCN2, which regulates cellular responses to amino acid availability by phosphorylating the translation initiation factor eIF2a. Phosphorylation of eIF2a reduces protein synthesis and induces expression of a transcription factor called ATF4. It is unclear if BMP and GCN2 signalling are functionally related and so we set out to test this using the model organism Drosophila melanogaster.

Methods We examined the development of wing veins in Drosophila because this is known to depend on BMP signalling. We manipulated the expression of dGCN2 and its antagonistic phosphatase dPPP1R15 by RNA interference. Morphology of the wing veins was examined in adult flies and early developmental signalling was measured in pupating animals. Similar experiments performed in animals with defective BMP signalling tested for a genetic interaction between eIF2a phosphorylation and BMP signalling.

Results We observed that depletion of dGCN2 in the developing wing by RNA interference enhanced BMP signalling as evidenced by hyperphosphorylation of MAD, the Drosophila orthologue of SMAD1. This was associated with development of ectopic wing veins in the regions of RNAi expression. In contrast, knock down of the eIF2a phosphatase dPPP1R15 selectively impaired anterior crossvein formation. The effects of dPPP1R15 depletion were cell-autonomous suggesting an effect on downstream BMP signalling rather than on ligand secretion. The vein phenotypes were highly sensitive to manipulation of BMP signalling. Both in insect and mammalian cells, ATF4 expression inhibited BMP signalling 